\title{
Design Optimization and Reliability Estimation with Incomplete Uncertainty Information
}

Subroto Gunawan, Michael Kokkolaras and Panos Y. Papalambros University of Michigan

Zissimos P. Mourelatos Oakland University

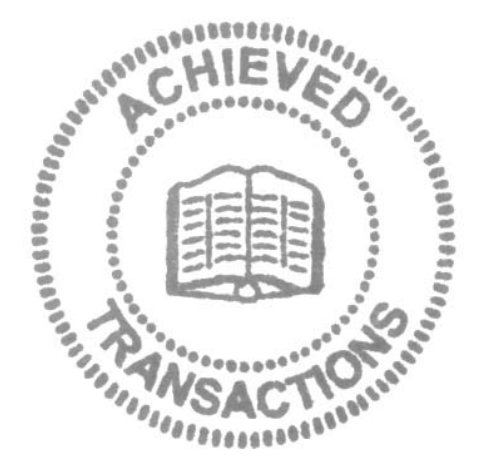

Reprinted From: Reliability and Robust Design in Automotive Engineering 2006

(SP-2032)

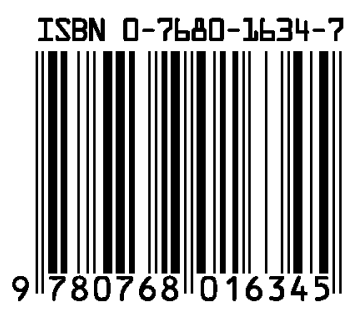


The Engineering Meetings Board has approved this paper for publication. It has successfully completed SAE's peer review process under the supervision of the session organizer. This process requires a minimum of three (3) reviews by industry experts.

All rights reserved. No part of this publication may be reproduced, stored in a retrieval system, or transmitted, in any form or by any means, electronic, mechanical, photocopying, recording, or otherwise, without the prior written permission of SAE.

For permission and licensing requests contact:

SAE Permissions
400 Commonwealth Drive
Warrendale, PA 15096-0001-USA
Email: permissions@ @ sae.org
Tel: $\quad 724-772-4028$
Fax: $\quad 724-776-3036$

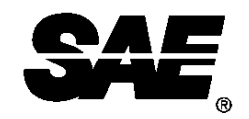

Global Mobility Database ${ }^{\circledast}$

All SAE papers, standards, and selected books are abstracted and indexed in the Global Mobility Database.

For multiple print copies contact:

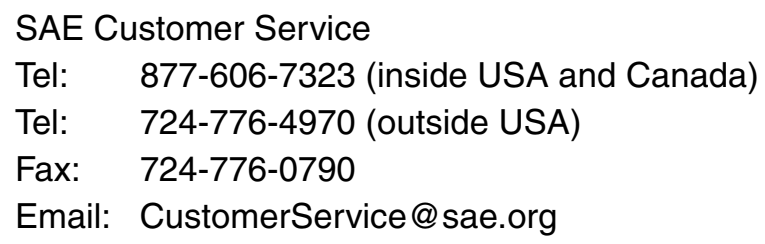

\section{ISSN 0148-7191}

\section{Copyright $\odot 2006$ SAE International}

Positions and opinions advanced in this paper are those of the author(s) and not necessarily those of SAE. The author is solely responsible for the content of the paper. A process is available by which discussions will be printed with the paper if it is published in SAE Transactions.

Persons wishing to submit papers to be considered for presentation or publication by SAE should send the manuscript or a 300 word abstract to Secretary, Engineering Meetings Board, SAE.

\section{Printed in USA}




\title{
Design Optimization and Reliability Estimation with Incomplete Uncertainty Information
}

\author{
Subroto Gunawan, Michael Kokkolaras and Panos Y. Papalambros \\ University of Michigan \\ Zissimos P. Mourelatos \\ Oakland University
}

Copyright $@ 2006$ SAE International

\begin{abstract}
Existing methods for design optimization under uncertainty assume that a high level of information is available, typically in the form of data. In reality, however, insufficient data prevents correct inference of probability distributions, membership functions, or interval ranges. In this article we use an engine design example to show that optimal design decisions and reliability estimations depend strongly on uncertainty characterization. We contrast the reliability-based optimal designs to the ones obtained using worst-case optimization, and ask the question of how to obtain nonconservative designs with incomplete uncertainty information. We propose an answer to this question through the use of Bayesian statistics. We estimate the truck's engine reliability based only on available samples, and demonstrate that the accuracy of our estimates increases as more samples become available. Finally, we use this information-based reliability assessment to optimize the engine while maximizing the confidence that the design will meet or exceed a prespecified reliability target.
\end{abstract}

\section{INTRODUCTION}

Deterministic design optimization does not account for uncertainties that can affect system performance during its lifetime and may come from various sources: modeling approximations, manufacturing imperfections, or unknown operating conditions. Due to these uncertainties, deterministic optimization may result in designs with poor reliability. To ensure that optimal designs are also reliable, the design optimization must account for uncertainties.

Reliability assessment, however, strongly depends on the choice of representation for the uncertain quantities. A popular approach is to quantify them probabilistically as random variables. However, this quantification assumes that a high level of information is available in the form of probability distribution functions (PDFs). In many cases this assumption is not realistic; insufficient data prevents correct inference of PDFs. Another approach is to use intervals to represent the uncertainty quantity. However, this approach is a worst-case scenario-based approach that yields conservative results. In practical design, information about uncertain quantities is typically available as finite collections of samples; for instance, from historical data, laboratory simulation, or field measurements. This representation constitutes incomplete information regarding the uncertain quantities. The challenge is then how to obtain an optimal and reliable design using this incomplete uncertainty information appropriately without having to resort to worst-case scenario analysis.

The rest of the article is organized as follows. In the following section we use a hydraulic-hybrid powertrain engine example to demonstrate design optimization using two uncertainty representations: probabilistic and interval. We show that the optimal and reliable design is sensitive to different representations. Next we demonstrate the use Bayesian statistics to estimate the engine's reliability based on finite samples. We then use this information-based reliability assessment to obtain the optimal and reliable engine design. The article is concluded with a brief summary.

\section{ENGINE OPTIMIZATION UNDER UNCERTAINTY}

Our design example considers the engine of a hybridized powertrain for a medium-sized truck. Previous efforts for improving fuel economy of medium trucks have shown that hybrid-hydraulic configurations could yield fuel economy improvements of up to $47 \%$ for a delivery truck [1] and $32 \%$ for a larger $6 \times 6$ off-road tactical truck [2].

The engine design optimization problem of the hydraulichybrid medium-sized truck is formulated as follows: 


\section{Given maximize design parameters with respect to subject to fuel economy \\ engine design variables satisfying performance constraints}

The objective is to maximize fuel economy (measured in miles per gallon) subject to three performance constraints: $0-45 \mathrm{mph}$ acceleration time should not exceed 24 seconds, and maintain a maximum speed of $55 \mathrm{mph}$ and $45 \mathrm{mph}$ on $2 \%$ and $3 \%$ grade, respectively. Preliminary studies have indicated that the acceleration constraint dominates the maximum speed constraints, so in this article we will focus on the former.

The baseline $6 \times 6$ all-wheel-drive truck is powered by a $246 \mathrm{KW}$ six-cylinder, turbocharged, intercooled, direct injection diesel engine and has a gross vehicle weight of $15,300 \mathrm{~kg}$. A detailed description of the truck model (including the embedded hydraulic system) is given in [3]. VESIM, a high fidelity vehicle/engine simulation environment [4], is used to evaluate fuel economy and the performance constraints. The driving cycle used for the simulation is depicted in Figure 1. The total simulation real-time amounts to 2200 seconds, and corresponds to a distance of approximately $25 \mathrm{~km}$ on a network of primary, secondary, and cross-country roads.

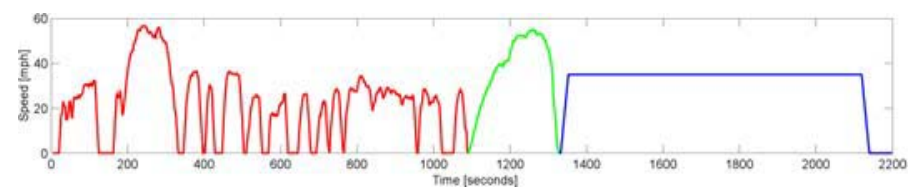

Figure 1. Driving cycle

The input list of the integrated VESIM simulation model includes a large number of quantities related to the hybrid powertrain and vehicle dynamics. To optimize the baseline, the designer has to decide which of those inputs to consider as design variables. The rest are held fixed to the baseline values and are considered as parameters. In this study we focus on the engine design. Although the hydraulic system design affects the performance of the hybrid powertrain, it is not included in this optimization study because the acceleration constraint must be satisfied assuming that only the engine is driving the vehicle. Therefore, we use the optimal hydraulic system design computed in a previous deterministic optimization study. Four engine design variables are selected to represent the engine design, and four parameters with the highest impact on the truck's fuel economy and performance are identified [5]. The bounds of the design variables and the nominal values of the design parameters are summarized in Table 1. They will all be considered uncertain in the following design optimization and reliability assessment case study.
Table 1. Design variables and parameters

\begin{tabular}{|l|c|c|}
\hline \multicolumn{1}{|c|}{ Design variable } & $\begin{array}{r}\text { Lower } \\
\text { bound }\end{array}$ & $\begin{array}{c}\text { Upper } \\
\text { bound }\end{array}$ \\
\hline Displacement (L) & 6 & 9 \\
\hline Compression ratio (-) & 12 & 17 \\
\hline Boost pressure (bar) & 1.87 & 2.55 \\
\hline Wastegate activation speed (RPM) & 1000 & 1600 \\
\hline \multicolumn{1}{|c|}{ Design parameter } & \multicolumn{2}{|c|}{ Nominal value } \\
\hline Injection timing ( ${ }^{\circ}$ ATDC) & \multicolumn{2}{|c|}{350} \\
\hline Frontal area (m ${ }^{2}$ ) & \multicolumn{2}{|c|}{7.5} \\
\hline Transmission efficiencies (-) & \multicolumn{2}{|c|}{0.94} \\
\hline $\begin{array}{l}\text { Differential and rolling resistance } \\
\text { scaling parameters (-) }\end{array}$ & \multicolumn{2}{|c|}{1.0} \\
\hline
\end{tabular}

In the presence of uncertainty, the design optimization problem must be modified. Next we optimize the engine design using two representations of the uncertain quantities (variables and parameters): probabilistic and interval.

\section{PROBABILISTIC APPROACH}

In this approach we assume that we have sufficient information and data to associate the uncertain quantities with probability distributions. Uncertain design variables and parameters are then modeled as random variables. Terminology becomes somewhat tricky: for simplicity, we will use the terms random design variables and random design parameters to distinguish between uncertain optimization variables and parameters that are modeled as random variables.

The probabilistic design optimization problem of the truck's engine is formulated as

\begin{tabular}{|c|c|}
\hline Given & $\begin{array}{l}\text { probability density functions of } \\
\text { random design variables and } \\
\text { random design parameters }\end{array}$ \\
\hline $\begin{array}{l}\text { maximize } \\
\text { with respect to }\end{array}$ & $\begin{array}{l}\text { expected value of fuel economy } \\
\text { mean values of random design } \\
\text { variables }\end{array}$ \\
\hline subject to & $\begin{array}{l}\text { probability of satisfying performance } \\
\text { constraints is greater than some value }\end{array}$ \\
\hline
\end{tabular}

Mean values of random design variables are chosen as variables in this optimization problem, and a first order approximation around the mean values of the random design variables and parameters is used for the expected values of the fuel economy. This problem can be solved using many appropriate methods to yield an optimal design that corresponds to the required reliability level specified by the designers $[6,7]$. We use the so- 
called single-loop method [8], an efficient method to solve reliability-based optimization problems.

In [5], we considered the case where all random variables are assumed to be normally distributed. The standard deviations of the random design variables and the means and standard deviations of the random design parameters were identified with the help of the simulation model developers, and are summarized here in Tables 2 and 3 . The probabilistic design problem was solved and the results were reported. In this article we assess the impact of incomplete information on design decisions.

Table 2. Standard deviations for random design variables

\begin{tabular}{|l|c|}
\hline \multicolumn{1}{|c|}{ Random Design Variable } & $\boldsymbol{\sigma}$ \\
\hline Displacement (L) & 0.025 \\
\hline Compression ratio (-) & 0.1 \\
\hline Boost pressure (bar) & 0.1 \\
\hline Wastegate activation speed (RPM) & 50 \\
\hline
\end{tabular}

Table 3. Means and standard deviations for random design parameters

\begin{tabular}{|l|c|c|}
\hline \multicolumn{1}{|c|}{ Random Design Parameter } & $\mu$ & $\sigma$ \\
\hline Injection timing ( ${ }^{\circ}$ ATDC) & 350 & 1 \\
\hline Frontal area $\left(\mathrm{m}^{2}\right)$ & 7.5 & 0.75 \\
\hline Transmission efficiencies (-) & 0.94 & 0.02 \\
\hline $\begin{array}{l}\text { Differential and rolling resistance } \\
\text { scaling parameters (-) }\end{array}$ & 1.0 & 0.1 \\
\hline
\end{tabular}

Specifically, let us assume that the knowledge or information used in [5] to infer normally distributed random variables was insufficient, and let us hypothesize that they are all, say, uniformly distributed. To keep things relatively comparable, let us define the uniform distribution parameters of the random variables such that the ranges of attainable values correspond to the $\mu \pm 2 \sigma$ ranges of the normally distributed variables. These parameters are summarized in Table 4.

Table 4. Uniform distribution parameters for random design variables and random design parameters

\begin{tabular}{|l|c|c|}
\hline \multicolumn{1}{|c|}{$\begin{array}{c}\text { Random Design Variable or } \\
\text { Parameter }\end{array}$} & a & b \\
\hline Displacement (L) & $\mu-0.05$ & $\mu+0.05$ \\
\hline Compression ratio (-) & $\mu-0.2$ & $\mu+0.2$ \\
\hline
\end{tabular}

\begin{tabular}{|l|c|c|}
\hline Boost pressure (bar) & $\mu-0.2$ & $\mu+0.2$ \\
\hline Wastegate activation speed (RPM) & $\mu-100$ & $\mu+100$ \\
\hline Injection timing ( $\left.{ }^{\circ} \mathrm{ATDC}\right)$ & 348 & 352 \\
\hline Frontal area (m $\left.{ }^{2}\right)$ & 6 & 9 \\
\hline Transmission efficiencies (-) & 0.90 & 0.98 \\
\hline $\begin{array}{l}\text { Differential and rolling resistance } \\
\text { scaling parameters (-) }\end{array}$ & 0.8 & 1.2 \\
\hline
\end{tabular}

The probabilistic problem was solved again and optimal designs were obtained for different reliability target values. There is not much value in reporting the obtained values of the design variables (the mean values of the random design variables) since it was expected that they were going to be different than the ones obtained assuming normally distributed random variables. Instead, we present the quantified tradeoffs between optimality and reliability in Figure 2 . The assumption of uniformly distributed random variables yields larger engine designs, which means less fuel economy. It is also interesting to see that the nonlinear behavior of the tradeoff curves is different.

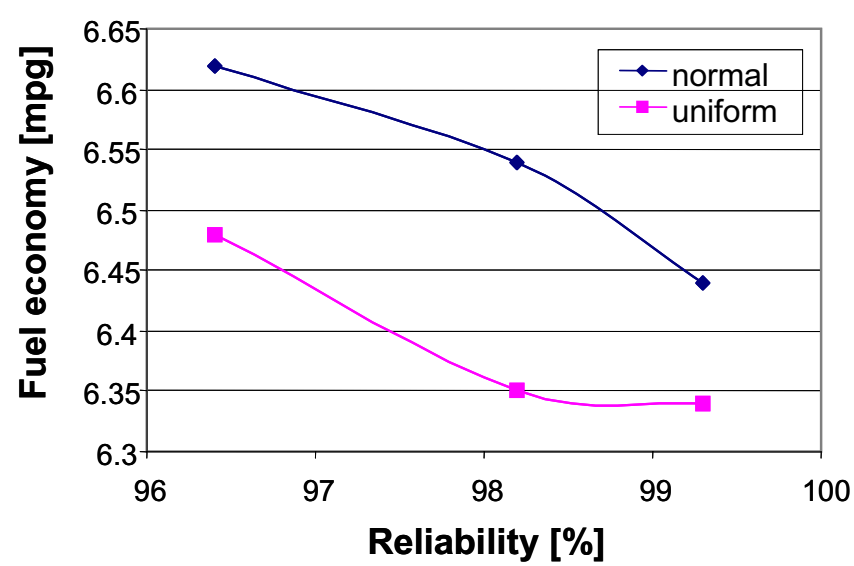

Figure 2. Nonlinear fuel economy-reliability tradeoffs

\section{INTERVAL APPROACH}

Let us now assume that we have very limited information or data, so that we cannot (or should not) infer probability distributions. Instead, we can only assume that the uncertain quantities can take any value within an interval. Note that this is not equivalent to assuming a uniform distribution as it does not imply that the probability of taking a specific value in a range is equal to any other value within that range. Mathematically, interval representation of the uncertain quantities is part of possibility theory [9]. In this case study we assume the lowest level of information with $\alpha=0$ corresponding to the widest interval of the uncertain quantities [10]. Tables 5 and 6 include the assumed intervals for the uncertain design variables and parameters. These 
intervals were chosen to be able to be contrasted with the ones of the probabilistic approach.

Table 5. Assumed uncertain design variable intervals

\begin{tabular}{|l|l|}
\hline \multicolumn{1}{|c|}{ Variable } & \multicolumn{1}{c|}{ Range } \\
\hline Displacement (L) & nominal \pm 0.05 \\
\hline Compression ratio (-) & nominal \pm 0.2 \\
\hline Boost pressure (bar) & nominal \pm 0.2 \\
\hline Wastegate activation speed (RPM) & nominal \pm 100 \\
\hline
\end{tabular}

Table 6. Assumed uncertain design parameter intervals

\begin{tabular}{|l|c|}
\hline \multicolumn{1}{|c|}{ Parameter } & Range \\
\hline Injection timing $\left({ }^{\circ}\right.$ ATDC) & $348-352$ \\
\hline Frontal area $\left(\mathrm{m}^{2}\right)$ & $6.0-9.0$ \\
\hline Transmission efficiencies (-) & $0.90-0.98$ \\
\hline $\begin{array}{l}\text { Differential and rolling resistance } \\
\text { scaling parameters (-) }\end{array}$ & $0.8-1.2$ \\
\hline
\end{tabular}

Using this interval representation for the uncertain quantities, the design optimization problem is modified as

$\begin{array}{ll}\text { Given } & \begin{array}{l}\text { intervals of design parameters } \\ \text { and intervals around nominal } \\ \text { design variable values }\end{array} \\ \text { maximize } & \text { minimal fuel economy } \\ \text { with respect to } & \text { nominal design variables } \\ \text { subject to } & \text { maximal acceleration time is less } \\ & \text { than 24 seconds }\end{array}$

Nominal values of the interval design variables are used as variables in the optimization problem. The objective is to maximize the worst-case value of the fuel economy while ensuring that the worst-case acceleration time satisfies the requirement. These worst-case values are obtained by solving two inner-loop optimization problems (also called "anti-optimization" problems [11]) so that the overall design optimization problem is a nested problem (double-loop). The DIRECT optimization algorithm [12] is used to solve this double-loop problem to maximize the likelihood of converging to global optima for both the outer and inner problems. Table 7 reports the obtained optimal engine design nominal values. More cases and detailed results are presented in [13, 14].
Table 7. Optimal design nominal values obtained using interval representation of uncertain variables and parameters

\begin{tabular}{|l|c|}
\hline \multicolumn{1}{|c|}{ Uncertain Design Variable } & $\begin{array}{c}\text { Optimal nominal } \\
\text { value }\end{array}$ \\
\hline Displacement (L) & 7.57 \\
\hline Compression ratio (-) & 16.77 \\
\hline Boost pressure (bar) & 2.34 \\
\hline Wastegate activation speed (RPM) & 1226 \\
\hline
\end{tabular}

The optimal design has been obtained considering the worst-case scenarios for both the fuel economy and acceleration time. To obtain their best-case scenario values, and thus the complete ranges of fuel economy and acceleration time within the intervals, the problem is solved one more time with reversed inner-loop objectives (maximal fuel economy and minimal acceleration time). Table 8 includes the intervals obtained for fuel economy and acceleration time and the associated variable and parameter values.

The information in Table 8 can be quite useful to the designer. For example, it can be seen that the same set of parameter values are adversary or beneficial to both fuel economy and acceleration time. Thus if the designers can allocate resources to reduce or control uncertainty, they know which parameter values to strive for. On the other side, it can be observed that engine displacement and wastegate activation speed extremes benefit or degrade either fuel economy or acceleration time, but not both simultaneously, while engine compression ratio and boost pressure extremes benefit or degrade fuel economy and acceleration time simultaneously.

Table 8. Obtained fuel economy and acceleration time intervals and associated variable and parameter values

\begin{tabular}{|l|c|c|c|c|}
\hline & $\begin{array}{c}\text { Min fuel } \\
\text { economy }\end{array}$ & $\begin{array}{c}\text { Max fuel } \\
\text { economy }\end{array}$ & $\begin{array}{c}\text { Min acc. } \\
\text { time }\end{array}$ & $\begin{array}{c}\text { Max acc. } \\
\text { time }\end{array}$ \\
\hline & $5.78 \mathrm{mpg}$ & $7.13 \mathrm{mpg}$ & $16 \mathrm{~s}$ & $24 \mathrm{~s}$ \\
\hline Displ. (L) & 7.62 & 7.52 & 7.62 & 7.52 \\
\hline Comp. ratio (-) & 16.57 & 16.97 & 16.97 & 16.57 \\
\hline Boost pres. (bar) & 2.14 & 2.54 & 2.54 & 2.14 \\
\hline $\begin{array}{l}\text { Waste. act. } \\
\text { speed (RPM) }\end{array}$ & 1126 & 1326 & 1126 & 1326 \\
\hline $\begin{array}{l}\text { Injection timing } \\
\left({ }^{\circ} \text { ATDC) }\right.\end{array}$ & 352 & 348 & 348 & 352 \\
\hline Front. Area (m $\left.{ }^{2}\right)$ & 9 & 6 & 6 & 9 \\
\hline
\end{tabular}




\begin{tabular}{|l|c|c|c|c|}
\hline $\begin{array}{l}\text { Transmission } \\
\text { efficiencies (-) }\end{array}$ & 0.90 & 0.98 & 0.98 & 0.90 \\
\hline $\begin{array}{l}\text { Rolling res./diff. } \\
\text { scaling (-) }\end{array}$ & 1.2 & 0.8 & 0.8 & 1.2 \\
\hline
\end{tabular}

\section{REMARKS ON THE PROBABILISTIC AND INTERVAL} APPROACHES

Although the probabilistic and interval approaches cannot be compared directly, we observe that the optimal design of the interval approach is very close to the optimal design of the probabilistic approach with a $99.3 \%$ reliability level. This demonstrates that optimal designs obtained using the interval approach tend to be conservative. It is also interesting to note that the approximate expected fuel economy value in the probabilistic approach at $99.3 \%$ reliability $(6.45$ and 6.35 mpg when assuming normally and uniformly distributed random variables, respectively) is very close to the midpoint of the fuel economy interval in the interval approach.

The interval approach provides a range that bounds the possible attainable values of the fuel economy while the probabilistic approach yields a single value, namely the approximate expected value of the fuel economy. Similarly, the probabilistic approach assures that design constraints will not be violated with a specific amount of confidence associated with prespecified reliability levels, while the interval approach excludes the possibility of constraint violation at the expense of a more conservative design. Moreover, it provides the range of attainable acceleration time values.

Since the interval approach yields a conservative optimal and reliable design, it may be thought that the probabilistic approach is preferred. However, this is the case only if we have a relatively large amount of data to properly infer PDF's of the uncertain quantities. In practical situations, the amount of data available is typically limited to a handful of samples due to cost or time constraints. Constructing a PDF from a small set of data should be avoided since it may result in highly erroneous analysis. The challenge is then how to design an optimal and reliable engine using this limited data sample without having to resort to either conservative worst-case analysis or inferring PDF's.

\section{FINITE SAMPLES APPROACH}

If we have sufficient data to infer PDF's of the engine's uncertain variables and parameters, we can calculate performance reliability of the engine design precisely as a single value. When data is limited, however, then it is not possible to assess reliability of a design with such precision. Instead, we can only calculate a distribution of the performance reliability in which some values may be more likely than the others. Precision of this distribution estimate depends on the number of data samples available: the larger it is, the better the reliability estimate becomes. This correlation can be calculated mathematically using Bayesian statistics [155, 166].

For this portion of the case study we assume that there are not sufficient data to infer PDF's of the displacement and compression ratio design variables. Instead, $N$ finite samples of their random values are collected through simulations and measurements. The other design variables and parameters are modeled as normally distributed random variables (according to Tables 2 and 3 ). As a demonstration, Figure 3 shows the change in a reliability estimate of a nominal engine design as the number of data samples increase. The design variable values of the nominal engine under consideration are: displacement $=6 \mathrm{~L}, \quad$ compression ratio $=17$, boost pressure $=2.55$ bar, and wastegate activation speed $=1200$ RPM. Figure 4 shows the $95 \%$ credibility interval of the estimate for different values of $N$.

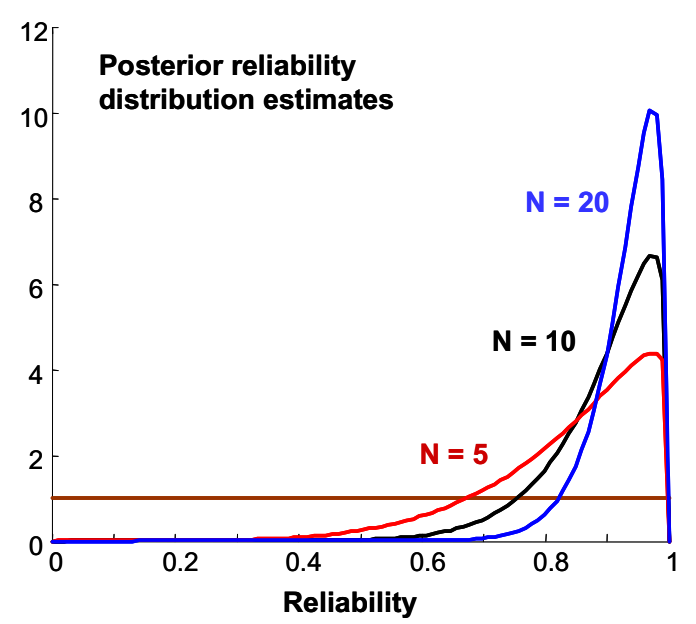

Figure 3. Improved reliability estimate with increasing data
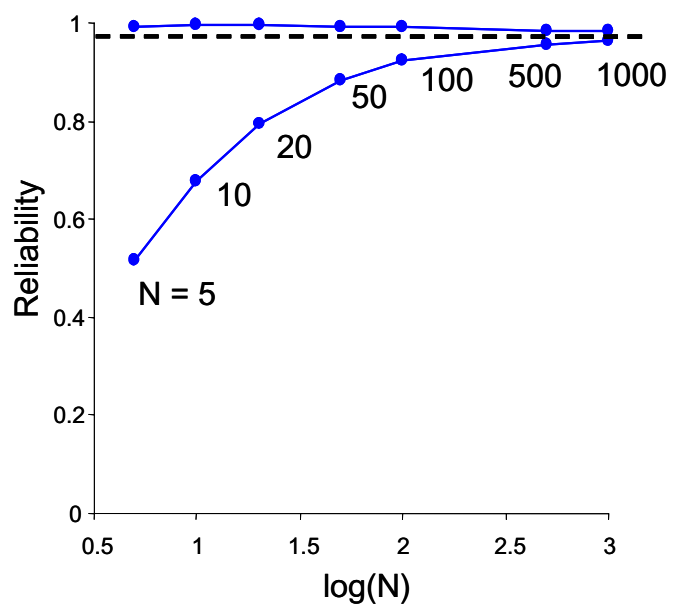

Figure $4.95 \%$ credibility interval of the reliability estimate

We see in Figures 3 and 4 that the reliability estimate of the nominal engine design for $N=5$ is rather poor. With this low number of available samples, reliability of the engine is estimated with $95 \%$ credibility to be between 0.5 and 1.0. As the number of samples increase, however, the reliability estimate becomes more precise. 
For $N=10$ and 20 , the $95 \%$ credible interval of the estimate reduces to within $[0.65,1.0]$ and $[0.8,1.0]$, respectively. For a very large number of sample $(N=$ 1000 ), we see that the reliability estimate converges to a single value of 0.97 . This is the actual reliability of this engine design.

Because we can only obtain a distribution of reliability estimates for an engine design, in the design optimization it is not possible to determine with certainty whether or not an engine satisfies the reliability threshold specified by the designers. Rather, we can only calculate the probability that reliability of an engine will meet or exceed the threshold value. This probability is called the confidence in the design. Using this confidence measure, the design optimization problem of the hydraulic-hybrid medium-sized truck is formulated as
Given
finite samples of the random design variables and parameters
maximize maximize with respect to expected value of fuel economy confidence in design's reliability random design variables

Notice that the design optimization problem now has two objectives: one is to maximize the expected value of the fuel economy, and the other is to maximize the confidence that the optimal design will satisfy the reliability threshold. Solving this optimization problem will in general result in a set of optimal engine designs, instead of just one optimal design. The maximum attainable confidence of the optimal designs also depends on the level of available data, i.e., the number of samples. It increases as $N$ increases. Figure 5 shows the optimal engine designs of the truck for different number of $N$. The threshold reliability value for this particular design optimization problem is specified to be 0.95 .

As can be seen in Figure 5, there is a tradeoff between fuel economy of an optimal engine design and confidence in its reliability. For all values of $N$, the highest fuel economy that can be achieved is $6.82 \mathrm{mpg}$. However, the confidence of this optimal design is zero, indicating that this particular engine design does not meet the specified 0.95 reliability level. As the confidence in the optimal design's reliability increases, the fuel economy decreases. Another important observation from Figure 5 is that the maximum attainable confidence of the optimal designs increases as $N$ increases. For $N=5$, the maximum confidence is rather low at 0.26; however, it increases to $0.43,0.66$, and 0.99 for $N=10,20$, and 100 , respectively. It is also seen that approximately 100 data samples are needed in this optimization problem to achieve nearly $100 \%$ confidence in the optimal engine design's reliability.
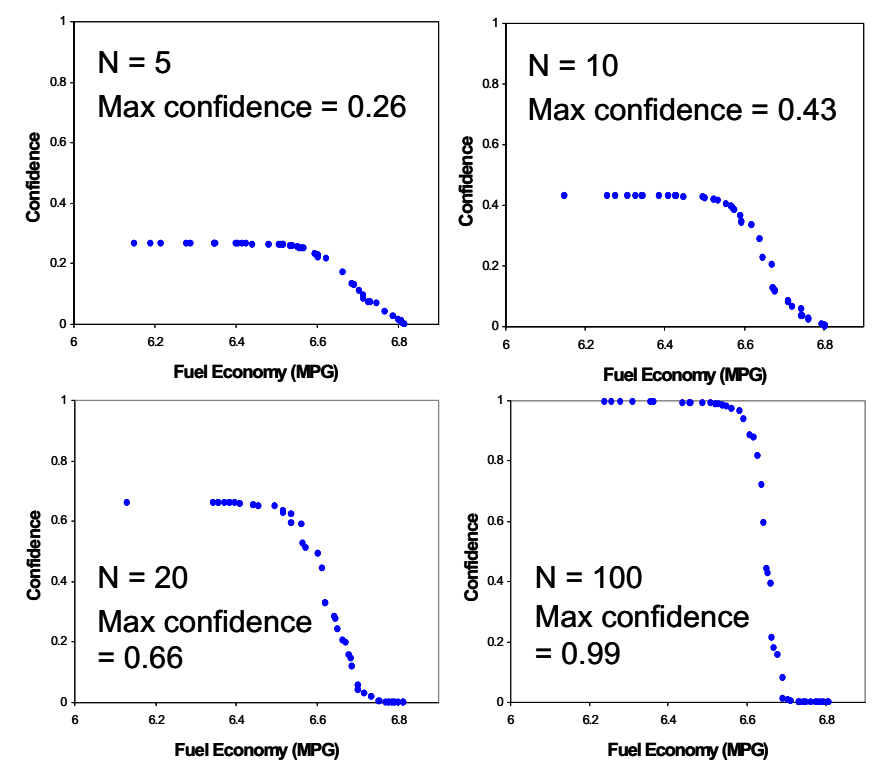

Figure 5. Confidence-optimality tradeoffs for increasing $N$

\section{SUMMARY}

In this article we have shown how uncertainties impact the design decisions for a hybrid-hydraulic engine optimization. Failing to account for uncertainties yields optimal designs associated with poor reliability. Moreover, accuracy of reliability assessments depends on the characterization of the uncertain design variables and parameters, which in turn depends on available information.

If a sufficiently high level of information is available (e.g., in form of data), then probabilistic representation provides the most complete picture of an engine design's reliability. In practice, however, data regarding the uncertain quantities is usually limited due to cost or time constraints and can lead to inferring erroneous probability distributions. In this situation, interval representation should be used to obtain the worst- and best-case scenarios of an engine's fuel economy and performance. However, worst-case optimization typically results in conservative designs. One way to bridge this gap is to characterize the uncertain quantities as finite samples, and then use Bayesian statistics to get an estimate of the engine's reliability. A very useful feature of this approach is that it provides a direct link between the precision of the reliability estimate and the level of available information. As the number of samples increases, the estimate becomes more precise. When used to optimize the hydraulic-hybrid truck's engine, this approach yields a set of optimal designs which trade off between fuel economy of the engine and the confidence that the optimal engine design meets the specified reliability target values. 


\section{ACKNOWLEDGMENTS}

The authors gratefully acknowledge the support of the Automotive Research Center (ARC), a U.S. Army Center of Excellence in Modeling and Simulation of Ground Vehicles at the University of Michigan. The first three authors also appreciate the support of the NSF through the grant award DMI-0503737. Such support does not constitute an endorsement by the funding agencies of the opinions expressed in the article.

\section{REFERENCES}

1. B. Wu, C. C. Lin, Z. Fillipi, H. Peng, and D. Assanis, "Optimal Power Management for a Hydraulic Hybrid Delivery Truck," Journal of Vehicle System Dynamics, 42(1-2):23-40, 2004.

2. Z. Fillipi, L. Louca, B. Daran, C. C. Lin, U. Yildir, B. Wu, M. Kokkolaras, D. Assanis, H. Peng, P. Papalambros, and J. Stein, "Combined Optimization of Design and Power Management of the Hydraulic Hybrid Propulsion System for a 6x6 Medium Truck," Int. Journal of Heavy Vehicle Systems, 11(3-4):371-401, 2004.

3. Z. Fillipi, L. Louca, A. Stephanopoulou, J. Pukrushpan, B. Kittirungsi, and H. Peng, "Fuel Cell APU for Silent Watch and Mild Electrification of a Medium Tactical Truck," Proc. of the SAE World Congress, March 2004, Detroit, MI, SAE 2004-011477.

4. D. Assanis, Z. Fillipi, S. Gravante, D. Grohnke, X. Gu, L. Louca, G. Rideout, J. Stein, and Y. Wang, "Validation and Use of SIMULINK Integrated, High Fidelity, Engine-In-Vehicle Simulation of the International Class VI Truck," Proc. of the SAE World Congress, March 2000, Detroit, MI, SAE 2000-01-0288.

5. M. Kokkolaras, Z. Mourelatos, L. Louca, Z. Fillipi, G. Delagrammatikas, A. Stephanopoulou, P. Papalambros, and D. Assanis, "Design Under Uncertainty and Assessment of Performance Reliability of a Dual-Use Medium Truck with Hydraulic-Hybrid Powertrain and Fuel Cell Auxiliary
Power Unit," Proc. of the SAE World Congress, April 2005, Detroit, MI, SAE 2005-01-1396.

6. X. Du and W. Chen, "Sequential Optimization and Reliability Assessment Method for Efficient Probabilistic Design," ASME Journal of Mechanical Design, 126(2):225-233, 2004.

7. J. Royset, A. Der Kiureghian, and E. Polak, "Reliability-Based Optimal Structural Design by the Decoupling Approach," Reliability Engineering \& Systems Safety, 73:213-221, 2001.

8. J. Liang, Z. Mourelatos, and J. Tu, "A Single-Loop Method for Reliability-Based Design Optimization," Proc. of the ASME IDETC, Sept. 2004, Salt Lake City, UT, DAC-27255.

9. D. Dubois and H. Prade, Possibility Theory, Plennum Press, New York, 1988.

10. T. J. Ross, Fuzzy Logic with Engineering Applications, McGraw Hill, New York, 1995.

11. I. Elishakoff, R. Haftka, and J. Fang, "Structural Design under Bounded Uncertainty - Optimization with Anti-optimization," Int. Journal of Computers \& Structures, 53(6):1401-1405, 1994.

12. D. Jones, "The DIRECT Global Optimization Algorithm," Encyclopedia of Optimization, 1:431-440, 2001.

13. M. Kokkolaras, Z.P. Mourelatos, and P.Y. Papalambros, "Impact of Uncertainties on Design Decisions for a Hydraulic-Hybrid Powertrain Engine," submitted to the 1st AIAA NonDeterministic Approaches Conference, May 1-4, 2006, Newport, Rhode Island.

14. M. Kokkolaras, Z.P. Mourelatos, and P.Y. Papalambros, "Impact of Uncertainty Quantification on Design: An Engine Optimization Case Study," submitted to the International Journal of Reliability and Safety.

15. P. Lee, Bayesian Statistics: An Introduction, Oxford University Press, New York, 1989.

16. P. Congdon, Bayesian Statistical Modeling, Wiley Series in Probability and Statistics, John Wiley \& Sons, New York, 2004. 